\title{
Measurement of gravitational time delay using drag-free spacecraft and an optical clock
}

\author{
Neil Ashby ${ }^{1}$, Peter L. Bender ${ }^{2,3}$, John L. Hall ${ }^{2,3}$, Jun Ye ${ }^{2,3}$, \\ Scott A. Diddams ${ }^{3}$, Steven R. Jefferts ${ }^{3}$, Nathan Newbury ${ }^{3}$, \\ Chris Oates $^{3}$, Rita Dolesi ${ }^{4}$, Stefano Vitale ${ }^{4}$, \& William J. Weber ${ }^{4}$ \\ ${ }^{1}$ University of Colorado, Boulder, CO \\ email: ashby@boulder.nist.gov \\ ${ }^{2}$ JILA, University of Colorado, Boulder, CO \\ ${ }^{3}$ National Institute of Standards \& Technology, Boulder, CO \\ ${ }^{4}$ University of Trento, Italy
}

\begin{abstract}
Improved accuracy in measurement of the gravitational time delay of electromagnetic waves passing by the sun may be achieved with two drag-free spacecraft, one with a stable clock and laser transmitter and one with a high-stability transponder. We consider one spacecraft near the Earth-Sun L1 point with an advanced optical clock, and the transponder on a second satellite, which has a 2 year period orbit and eccentricity $e=0.37$. Superior conjunctions will occur at aphelion 1, 3, and 5 years after launch of the second spacecraft. The measurements can be made using carrier phase comparisons on the laser beam that would be sent to the distant spacecraft and then transponded back. Recent development of clocks based on optical transitions in cooled and trapped ions or atoms indicate that a noise spectral amplitude of about $5 \times 10^{-15} / \sqrt{\mathrm{Hz}}$ at frequencies down to at least 1 microhertz can be achieved in spaceborne clocks. An attractive candidate is a clock based on a single laser-cooled $\mathrm{Yb}^{+}$trapped ion. Both spacecraft can be drag-free at a level of $1 \times 10^{-13} \mathrm{~m} / \mathrm{s}^{2} / \sqrt{\mathrm{Hz}}$ at frequencies down to at least 1 microhertz. The corresponding requirement for the LISA gravitational wave mission is $3 \times 10^{-15} \mathrm{~m} / \mathrm{s}^{2} / \sqrt{\mathrm{Hz}}$ at frequencies down to $10^{-4} \mathrm{~Hz}$, and Gravitational Reference Sensors have been developed to meet this goal. They will be tested in the LISA Pathfinder mission, planned by ESA for flight in 2011. The requirements to extend the performance to longer times are mainly thermal. The achievable accuracy for determining the PPN parameter $\gamma$ is about $1 \times 10^{-8}$.
\end{abstract}

Keywords. gravitation, relativity, time delay

\section{Introduction}

Testing general relativity (GR) more fully is one of the important scientific opportunities for new missions and new observation programs in the next decade. The extra gravitational time delay for two-way measurements of light propagating from Earth to a spacecraft passing behind the Sun can be more than 200 microseconds. In the Parametrized Post-Newtonian (PPN) formulation of gravitational theory, the main contribution to the time delay is proportional to $(1+\gamma)$, where $\gamma$ is a measure of the curvature of space-time. In GR, $\gamma=1$.

Recently, a measurement of $\gamma$ with accuracy $\pm 2.3 \times 10^{-5}$ was made during the Cassini mission (Bertotti et al. (2003)). Further improvements in the accuracy for $\gamma$ to roughly $10^{-6}$ are expected from two missions of the European Space Agency (ESA): the Gaia astrometric mission, which will measure the gravitational deflection of light rays by the 
Sun, and the BepiColombo mission to Mercury, which will make improved measurements of the solar time delay.

Analysis of a time delay mission involving an accurate clock in a spacecraft at L1 began in 2005 (Ashby \& Bender (2008)). This proposal was updated recently to include the use of extremely stable new clocks based on optical transitions in cooled atoms or ions (Bender, et al.(2008)). We describe here a mission that can reach an accuracy of about $1 \times 10^{-8}$ for determining $\gamma$.

\section{Science justification}

In view of the well-known lack of a theory that connects general relativity with quantum theory, improvement of high-accuracy tests of the predictions of GR should be the object of research in the coming decade. Many alternatives to general relativity involve additional scalar fields. Studies of the evolution of scalar fields in the matter-dominated era of the universe indicate that the universe's expansion tends to drive the scalar fields toward a state in which the scalar-tensor theory is only slightly different from GR. Some scalar-tensor extensions of GR predict deviations from the GR value of $\gamma$ in the range from $10^{-5}-10^{-8}$ (Damour \& Esposito-Farese (1996), Damour \& Nordvedt (1993)). Improved information about $\gamma$ would provide important insight into the evolution of the universe and directly limit the range of applicability of alternative gravitational theories.

\section{Mission orbits and predicted time delay}

For the proposed mission, one spacecraft (S1) containing a highly stable optical clock would be placed in an orbit near the L1 point, about 1.5 million $\mathrm{km}$ from the Earth in the direction of the Sun. The second spacecraft (S2) would be placed in a 2 year period orbit in the ecliptic plane, with an eccentricity of 0.37. S2 would pass through superior solar conjunction about 1, 3, and 5 years after launch and would be near aphelion at those times. Both spacecraft would have drag-free systems to nearly eliminate the effects of spurious non-gravitational forces. A measurement of $\gamma$ to a level of $1 \times 10^{-8}$ would be carried out by observing the time delay of laser signals exchanged between the two spacecraft when the line of sight passed near the Sun's limb. Atmospheric effects would be absent and continuous observation would be possible. With S2 near aphelion, the range rate would be low, and the orbit determination problem would be much reduced.

The crucial measurements of time delay occur within a few days of superior conjunction and are primarily characterized by a logarithmic dependence on the distance of closest approach of the light to the mass source. The predicted gravitational time delay due to a non-rotating mass source (here $\mu=G M_{\odot} / c^{2}$ ), expressed in terms of the radii $r_{A}, r_{B}$ of the endpoints of the photon path, and the elongation angle $\Phi$ between the radius vectors from the source to the endpoints, is (Ashby \& Bertotti (2009))

$$
\begin{array}{r}
c \Delta t_{\text {delay }}=\mu(1+\gamma) \log \left(\frac{r_{A}+r_{B}+r_{A B}}{r_{A}+r_{B}-r_{A B}}\right)-\frac{\mu^{2}(1+\gamma)^{2} r_{A B}}{r_{A} r_{B}(1+\cos \Phi)} \\
+\frac{\mu^{2} r_{A B}(8-4 \beta+8 \gamma+3 \epsilon)}{4 r_{A} r_{B} \sin \Phi}
\end{array}
$$

where $r_{A B}$ is the geometric distance between the endpoints in isotropic coordinates and $\beta$ and $\epsilon$ are PPN parameters measuring the nonlinearity of the time-time and spacespace components of the metric tensor. In GR, $(8-4 \beta+8 \gamma+3 \epsilon) / 4=15 / 4$. The time delay in Eq. (3.1) is expressed in terms of observable quantities, and does not involve the 
unknown impact parameter or distance of closest approach. The non-linear terms are a few nanoseconds so are significant, but do not have to be estimated with great accuracy.

The contributions to time delay due to the solar quadrupole moment are controlled by the parameter $\mu J_{2} R^{2} /\left(b^{2} c\right)<10^{-12} \mathrm{~s}$, where $J_{2} \approx 2 \times 10^{-7}, R$ is the sun's radius, and $b \approx R$ is the impact parameter. This effect depends on the relative orientation of the sun's rotation axis and the photon path, but the net effect is small and can be estimated with sufficient accuracy that it will not contribute significantly to the error budget.

The measurements will be made by transmitting a continuous laser beam from S1 to $\mathrm{S} 2$, offset phase locking the laser signal from the transponder on the distant spacecraft to the received beam, and then recording the relative phase of the received beam back at S1 with respect to its own laser as a function of time. With $20 \mathrm{~cm}$ diameter telescopes, and given the one-way travel time of about $1600 \mathrm{~s}$, the received signal would be roughly 1000 counts/s for $1 \mathrm{~W}$ of transmitted power. This is a weak signal, but it is strong enough so that the chances of a cycle slip should be very small, provided that the laser in S2 is well stabilized. If we consider the round-trip delay times $\Delta t_{\text {delay }}$ to be the observable, then the change in delay from 0.75 days to 4 days on either side of conjunction is about 64 microseconds.

For initial acquisition of the beat frequency signal on S2, accurate knowledge of the orbits of both spacecraft is needed. Thus K-band tracking of both spacecraft from Earth would be needed over a period of 20 or 30 days around solar conjunction. In addition, a priori knowledge of the frequency of the laser on S2 to a kilohertz or so would be needed to simplify the acquisition procedure. As an example, if NdYAG lasers are used for transmission between the spacecraft, the stabilization of the one on S2 could be achieved by using a fairly simple iodine stabilization scheme. In this case, a frequency comb approach would be needed on S1 to tie the NdYAG laser frequency there to the highly stable optical clock frequency.

\section{Signal-to-Noise Analysis}

We estimate the uncertainty that can be attained in this experiment on the basis of the optimal Wiener filter, which takes advantage of the known time signature of the signal and includes the expected noise sources (Thorne (1987)). For simplicity, the distance between the spacecraft is assumed to be constant except for changes in the gravitational time delay. The time signature of $\gamma^{*}=(1+\gamma) / 2$ is taken to be represented by the logarithmic function

$$
g(t)=-B(\log |R t|-M)
$$

where $M$ is the mean value of $\log |R t|$ over the time periods $-t_{2}$ to $-t_{1}$ and $t_{1}$ to $t_{2}$ (a short time interval around occultation is excluded), and for the proposed experiment $B=0.97 \times 8 \mu / c=3.82 \times 10^{-5}$ s. The rate at which the line of sight to the distant spacecraft passes across the sun is $R=1.9$ solar radii per day.

Let $g(f)$ be the Fourier transform of $g(t)$ over the time of the measurements. Then the square of the signal-to-noise ratio is given by

$$
\left(\frac{S}{N}\right)^{2}=\int_{0}^{\infty} \frac{2|g(f)|^{2}}{n(f)^{2}} d f=\frac{2}{n(f)^{2}} \int_{0}^{\infty}|g(f)|^{2} d f
$$

where

$$
g(f)=2 \int_{t_{1}}^{t_{2}} g(t) \cos (2 \pi f t) d t,
$$

and the factor of 2 comes from time symmetry of the time-delay signal. Here we use a 


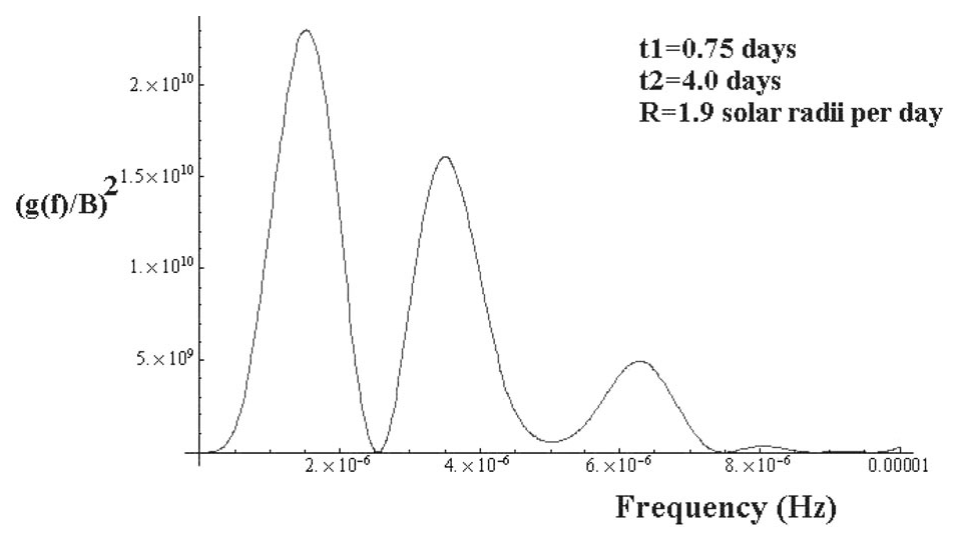

Figure 1. Plot of the function $g(f)^{2} / B^{2}$ for frequencies up to $0.01 \mathrm{mHz}$, for a typical set of measurement parameters.

one-sided Fourier transform, in which negative frequencies are folded into positive frequencies. The mean value $M$ is $M=\left(t_{2} \log \left(R t_{2}\right)-t_{1} \log \left(R t_{1}\right)\right) /\left(t_{2}-t_{1}\right)-1$. For example, for the case where the time interval of measurements extends from 0.75 days to 4 days, Figure 1 plots the quantity $g(f)^{2} / B^{2}$. When combined with estimates of the spectral density of the noise, the function gives us the signal to noise ratio according to Eq. (4.2) above. If the noise has a constant spectral density, only about $5 \%$ of the integral in Eq. (4.2) comes from frequencies below 1 microhertz, where the acceleration noise is expected to increase.

So far, we have assumed that time-delay measurements are only made over a total period of 8 days around solar conjunction. This was done in order to make sure that spurious acceleration noise at frequencies below $1 \times 10^{-6} \mathrm{~Hz}$ would have little effect. However, simulations for longer observing times are desirable, with full allowance for spurious acceleration noise at the lowest frequencies, as well as for the orbit determination part of the problem.

\section{Spacecraft S1 clock}

The major requirement for the mission is to fly an optical clock on S1 that has very high stability over a period of at least 8 days around superior conjunction. The nominal design goal for the mission is to achieve a fractional frequency noise power spectral density amplitude of $5 \times 10^{-15} / \sqrt{\mathrm{Hz}}$ from $1 \mathrm{~Hz}$ down to at least $10^{-6} \mathrm{~Hz}$. (This is nearly equivalent to an Allan deviation of $3.5 \times 10^{-15} / \sqrt{\tau}$ for times from $1 \mathrm{~s}$ to up to $10^{6} \mathrm{~s}$.) As an example of the desired performance at frequencies near $3 \mathrm{mHz}$, a spectral density amplitude of about $2 \times 10^{-15} / \sqrt{\mathrm{Hz}}$ has been achieved for the $698 \mathrm{~nm}$ transition in $\mathrm{Sr}$ atoms trapped in an optical lattice (Ludlow et al. (2008)).

A leading candidate for the optical clock is based on a single trapped cooled $\mathrm{Yb}^{+}$ion. The clock transition wavelength is $435 \mathrm{~nm}$. The projected spectral density amplitude is about $3 \times 10^{-15} / \sqrt{\mathrm{Hz}}$ over the necessary frequency range (Peik, E., et al. (2006)). An advantage of the $\mathrm{Yb}^{+}$clock is that only low power lasers are required. However, substantial development is needed to show that such lasers can be space qualified. Possible alternate choices include trapped ion clocks based on $\mathrm{Sr}^{+}$or $\mathrm{Al}^{+}$.

The relative phase of the transmitted and transponded signals would be strongly affected by the relative motion of the two spacecraft in their orbits, and thus all of the 
relevant orbit parameters as well as $\gamma$ would have to be solved for. With the S2 orbit considered here, the S1-S2 distance passes through a maximum at conjunction, when S2 is at aphelion. This reduces the effect of errors due to temperature fluctuations as well as measurement epoch errors. A worst-case drift of 0.1 ps in the clock that is correlated with the predicted time delay would give rise to an error of $3 \times 10^{-9}$ in determining $\gamma$.

In the time delay measurements, care will be needed to reduce the effects of scattered light in the telescopes. Because of the geometry, it would be difficult to avoid having some direct sunlight hit parts of the receiving optics. But, with heterodyne detection, this does not appear to be a serious problem. The plan for such a mission would be to not try to make measurements closer to the limb than about 0.4 solar radii, in order to avoid problems with reacquisition of the signals after solar conjunction.

\section{Drag-free system}

The required performance builds on that planned for the LISA mission. For frequencies down to $10^{-4} \mathrm{~Hz}$ for LISA, the requirement on the acceleration power spectral density amplitude is less than $3 \times 10^{-15} \mathrm{~m} / \mathrm{s}^{2} / \sqrt{\mathrm{Hz}}$. However, the performance is expected to degrade at lower frequencies. The main challenge for achieving good performance at low frequencies is minimizing thermal changes, and particularly thermal gradient changes, near the freely floating test mass in the drag-free system. On LISA this is done almost completely by passive thermal isolation. For a time delay mission, a fairly slow active temperature control system would be used at frequencies below $10^{-4} \mathrm{~Hz}$. Changes in solar heat input over the 8 days around conjunction would be quite small for $\mathrm{S} 2$, because conjunction occurs near aphelion. The required drag-free performance is roughly $1 \times$ $10^{-13} \mathrm{~m} / \mathrm{s}^{2} / \sqrt{\mathrm{Hz}}$ down to $10^{-6} \mathrm{~Hz}$.

In fact, much of the desired freedom from spurious accelerations needed for LISA has been demonstrated in the laboratory with torsion pendulum measurements (Carbone et al. (2007)). But, more important, the overall performance of the drag-free system will be demonstrated in the LISA Pathfinder Mission, which is scheduled for launch by ESA in 2011 (Armano et al. (2009); Racca \& McNamara (2009)). The requirements are just to demonstrate an acceleration spectral density amplitude of $3 \times 10^{-14} \mathrm{~m} / \mathrm{s}^{2} / \sqrt{\mathrm{Hz}}$ performance down to $10^{-3} \mathrm{~Hz}$, because of the less ideal thermal stability expected for LISA Pathfinder, compared with LISA. However, extensive tests of the response to various intentional thermal, electrical, magnetic, etc. disturbances will be carried out, in order to verify the models being used for the disturbances on the test masses. Thus the design of the drag-free systems for a gravitational time delay mission does not appear to be a substantial limitation.

\section{Other scientific benefits from the mission}

Additional effects such as those arising from non-linear terms in the 00-component of the metric tensor, parameterized by $\beta$, as well as other time delay effects originating in the sun's rotation, can also be measured. The clock at the L1 point will experience frequency shifts from the earth's potential, solar tidal effects, and second-order Doppler shifts. Relative to a reference on earth's surface, the fractional frequency shift is about $+6.9 \times 10^{-10}$, and is almost all gravitational. Comparing the clock at L1 with a similar clock on earth's geoid will give accuracies of a few parts per million in a few hours, which is orders of magnitude more accurate than the Vessot-Levine 1976 Gravity Probe A result. 


\section{Conclusion}

Measurement of the time delay of signals passing near the sun is a promising test of the predictions of GR that may provide two orders of magnitude improvement in determination of $\gamma$.

\section{References}

Armano, M. et al., 2009 in: LISA Pathfinder: the experiment and the route to LISA, Class. EG Quantum Grav. 26(9), 094001

Ashby, N. \& Bender, P. 2008, in: H. Dittus, C. Laemmerzahl, \& S. Turyshev, (eds.), Lasers, Clocks, and Drag-Free Control, Bremen, Germany, June 2005 Astrophysics and Space Science Library 349, (Springer) 219-230

Ashby, N. \& Bertotti, B. 2009, Accurate light-time correction due to a gravitating mass, to be submitted

Bertotti, B., Iess, L., \& Tortota, P. 2003, Nature, 425, 374

Bender, P. et al. 2008 in: Quantum to Cosmos III Workshop, Warrenton, VA, July 6-10.

Carbone, L. et al. 2007 Phys. Rev. D75(4), 042001

Damour, T. \& Esposito-Farese, G. 1996 Phys. Rev. D54, 5541

Damour, T. \& Nordtvedt, K. 1993 Phys. Rev. D48, 3436

Ludlow, A. D. et al. 2008 Science 399, 1805

Peik, E. et al. 2006 J. Phys. B39, 145-158

Racca, G. \& McNamara, P. 2009 Space Science Reviews in press

Thorne, K. S. 1987 Ch. 9 in: S. W. Hawking and W. Israel, eds. 300 Years of Gravitation (Cambridge University Press) 330-459 